\title{
Minimizing radiographic contrast administration during coronary angiography using a novel contrast reduction system: A multicenter observational study of the DyeVert ${ }^{\mathrm{TM}}$ plus contrast reduction system
}

\author{
Hitinder S. Gurm MD ${ }^{1} \odot$ | Kreton Mavromatis $\mathrm{MD}^{2}$ | Barry Bertolet $\mathrm{MD}^{3}$ | \\ Dean J. Kereiakes MD ${ }^{4}$ ( ) Amit P. Amin MD, MSc ${ }^{5}$ | Atman P. Shah MD ${ }^{6}$ | \\ George S. Hanzel MD ${ }^{7}$ | Siddhartha Rao MD ${ }^{8}$ | Joseph L. Thomas MD ${ }^{9}$ | Gautam Kumar MD²
}

${ }^{1}$ Department of Medicine, Division of Cardiovascular Medicine, University of Michigan, Ann Arbor, Michigan

${ }^{2}$ Department of Medicine, Cardiology Division, Atlanta VA Medical Center, Emory University School of Medicine, Decatur, Georgia

${ }^{3}$ Cardiology Associates Research, LLC, North Mississippi Medical Center, Tupelo, Mississippi

${ }^{4}$ Christ Hospital, Heart and Vascular Center, Lindner Research Center, Cincinnati, Ohio

${ }^{5}$ Cardiovascular Division, Washington University School of Medicine, St. Louis, Missouri

${ }^{6}$ Section of Cardiology, University of Chicago Medical Center, Chicago, Illinois

${ }^{7}$ Department of Medicine, Division of Cardiology, William Beaumont Hospital, Heart and Vascular, Royal Oak, Michigan

${ }^{8}$ WakeMed Heart and Vascular, Raleigh, North Carolina

${ }^{9}$ Division of Cardiology, Harbor-UCLA Medical Center, Torrance, California

Correspondence

Hitinder S. Gurm, MD, 2A 192F, Frankel

Cardiovascular Center, University of Michigan Health System, 1500 E Medical Center Drive, Ann Arbor, MI 48109-5853.

Email: hgurm@med.umich.edu

Funding information

Osprey Medical, Inc.

\begin{abstract}
Objective: To evaluate contrast media (CM) volume (CMV) saved using the DyeVert ${ }^{\mathrm{TM}}$ Plus Contrast Reduction System (DyeVert Plus System, Osprey Medical) in patients undergoing diagnostic coronary angiogram (CAG) and/or percutaneous coronary interventional ( $\mathrm{PCI}$ ) procedures performed with manual injections.

Background: Current guidelines advocate for monitoring and minimization of the total volume of $\mathrm{CM}$ in chronic kidney disease (CKD) patients undergoing invasive cardiac procedures. The DyeVert Plus System is an FDA cleared device designed to reduce CMV delivered during angiography and permit real-time CMV monitoring.

Methods: We performed a multicenter, single-arm, observational study. Eligible subjects were $\geq$ 18 years old with baseline estimated glomerular filtration rate (eGFR) $20-60 \mathrm{~mL} / \mathrm{min} / 1.73 \mathrm{~m}^{2}$. The primary endpoint was \% CMV saved over the total procedure. A secondary objective was to evaluate adverse events (AEs) related to DyeVert Plus System or to CM use.

Results: A total of 114 subjects were enrolled at eight centers. Mean age was $72 \pm 9$ years, $72 \%$ were male, and mean body mass index was $29 \pm 5$. Baseline eGFR was $43 \pm 11 \mathrm{~mL} / \mathrm{min} / 1.73$ $\mathrm{m}^{2}$. CAG-only was performed in $65 \%$ of cases. One hundred and five subjects were evaluable for the primary endpoint. Mean CMV attempted was $112 \pm 85 \mathrm{~mL}$ (range 22-681) and mean CMV delivered was $67 \pm 51 \mathrm{~mL}$ (range 12-403), resulting in an overall CMV savings of $40.1 \pm 8.8 \%$ (95\% $\mathrm{Cl} 38.4,41.8$; $P<0.0001)$ per procedure. Image quality was maintained in all but one case where the system was turned off for one injection. No DyeVert Plus System-related AEs were reported. Acute kidney injury (AKI; defined as serum creatinine rise of $>0.3 \mathrm{mg} / \mathrm{dL}$ from baseline) was reported in 11 cases with seven occurring in subjects with baseline eGFR $<30$ and three $\mathrm{AKI}$ events were attributed to CM. AKI rates increased as CMV/eGFR ratios increased.

Conclusions: These data suggest DyeVert Plus System use in CKD patients undergoing CAG and/or PCI results in clinically meaningful CMV savings while maintaining image quality.
\end{abstract}

\section{KEYWORDS}

ANCO-angiography, angiographic/fluoroscopic, CONT-contrast agents, coronary, IAFimaging, $\mathrm{PCl}$-percutaneous coronary intervention, RDAC-renal disease-acute 


\section{1 | INTRODUCTION}

Contrast-induced acute kidney injury (Cl-AKI) is a common complication observed in patients undergoing invasive cardiac procedures and is associated with increased morbidity, mortality, and health care costs. ${ }^{1-5}$ Current professional society recommendations support identification of at risk patients, appropriate periprocedural hydration, and minimization of contrast volume in at-risk patients as strategies to prevent $\mathrm{Cl}-\mathrm{AKI}{ }^{6,7}$

There appears to be a non-linear increase in risk of $\mathrm{AKI}$ with increasing doses of contrast media (CM) volume (CMV), and both in vivo data and clinical studies have demonstrated an association between high contrast volume and the risk of AKI., ${ }^{8,9}$ While different authors have evaluated several contrast thresholds to guide safe contrast dosing, collaborative efforts to reduce the proportion of patients exceeding threshold targets have been associated with a reduction in the incidence of $\mathrm{AKI} .{ }^{10-15}$

The DyeVert ${ }^{\mathrm{TM}}$ Contrast Reduction System (DyeVert System, Osprey Medical) was cleared by the Food and Drug Administration (FDA) for the purpose of reducing CMV delivered to patients during angiography procedures. Early experience with this device from European centers has demonstrated clinically and statistically meaningful reductions in CM delivered to the patient. ${ }^{16-18}$ We report the results of a single-arm, observational study evaluating the safety and efficacy of the latest version of the product, DyeVert Plus System, which includes continuous $\mathrm{CM}$ threshold monitoring, across multiple participating sites.

\section{2 | METHODS}

\section{1 | Study design and population}

This was a prospective, multicenter, single-arm, observational study designed to evaluate CMV saved using the DyeVert Plus System in a cohort of subjects undergoing diagnostic coronary angiography (CAG) and/or percutaneous coronary interventional $(\mathrm{PCI})$ procedures performed with manual injections. The study was performed at eight centers by 17 interventional cardiologists.

Local institutional review boards approved this study and all subjects provided written informed consent. Eligible subjects were $\geq 18$ years old, scheduled to undergo CAG and/or PCl, and had a baseline estimated glomerular filtration rate (eGFR) of $\geq 20$ and $\leq 60 \mathrm{~mL} /$ $\min / 1.73 \mathrm{~m}^{2}$. Subjects were excluded from participation if they: had acute ST-elevation myocardial infarction or known coronary artery fistulas, had a body mass index $(\mathrm{BMI})>40$, were currently pregnant, were undergoing a chronic total occlusion procedure or optical coherence tomography analysis, were planning to undergo transcatheter aortic valve replacement within $72 \mathrm{hr}$ of the index procedure, or had a condition known to require large volumes of contrast $(>10 \mathrm{~mL}$ ) for each injection.

The specific type of $\mathrm{CM}$, and the use of other renal protection strategies such as hydration, pre and post procedural laboratory studies, and continuation or discontinuation of specific medications were at the discretion of the study investigator and per local institutional policies. Similarly, the use of other contrast minimization techniques such as biplane angiography and use of adjunct imaging such as IVUS were per operator discretion and was not specified by the study protocol.

\subsection{Study device}

The DyeVert Plus System interfaces with standard manifold systems to provide real-time contrast monitoring and reduce the amount of contrast used in catheterization procedures while maintaining fluoroscopic image quality. System components include a disposable, singleuse, sterile DyeVert Plus Disposable Kit that contains a Smart Syringe and DyeVert Plus Module, which is connected to a standard manifold (Figure 1) and provides fluid pathway resistance modulation via a dedicated diversion valve. The diversion valve self-adjusts to the manual injection pressure to divert some of the CM into the reservoir chamber within the module. This diverted volume of CM does not enter the patient.

The second component of the system is a contrast monitoring wireless (CMW) display, which communicates with the DyeVert Plus disposable component to allow real-time monitoring and display of CMVs manually injected. The treating physician specified a maximum contrast dose threshold at their discretion prior to the procedure. This allowed determination of the percent of the predefined maximum CMV delivered to the patient. CM monitoring and volume accounting is completed via translation of Hall Effect sensors and pressure transducer voltage readings to volume readings in milliliters and accounting for whether contrast accounting for each injection was performed with the contrast accounting system on or off. At the end of the procedure, the $\mathrm{CMW}$ displays total procedure contrast volume used ( $\mathrm{mL}$, actual CMV delivered to the patient), \% of physician-specified threshold, total procedure contrast volume saved $(\mathrm{mL})$, and \% contrast saved. The amount of CMV attempted to be delivered to the patient is the sum of the total procedure contrast volume used $(\mathrm{mL})$ and total procedure contrast volume saved (mL).

Image quality was monitored by each operator during the procedure per standard practices. Any injection in which the DyeVert Plus System was turned off for the purpose of improving image quality was recorded.

\section{3 | Endpoints}

The primary endpoint of the study was the percentage of CMV saved over the total procedure as reported on the DyeVert Plus display at the end of each case.

Secondary endpoints were the evaluation of DyeVert Plus System-related adverse events (AEs) and CM-related AEs of anaphylaxis and AKI through discharge. Acute kidney injury events were defined as a $>0.3 \mathrm{mg} / \mathrm{dL}$ increase in serum creatinine postprocedure through discharge compared to the baseline value or through the date of a secondary procedure for staged procedures. Investigators defined the etiology of each AKI event. 


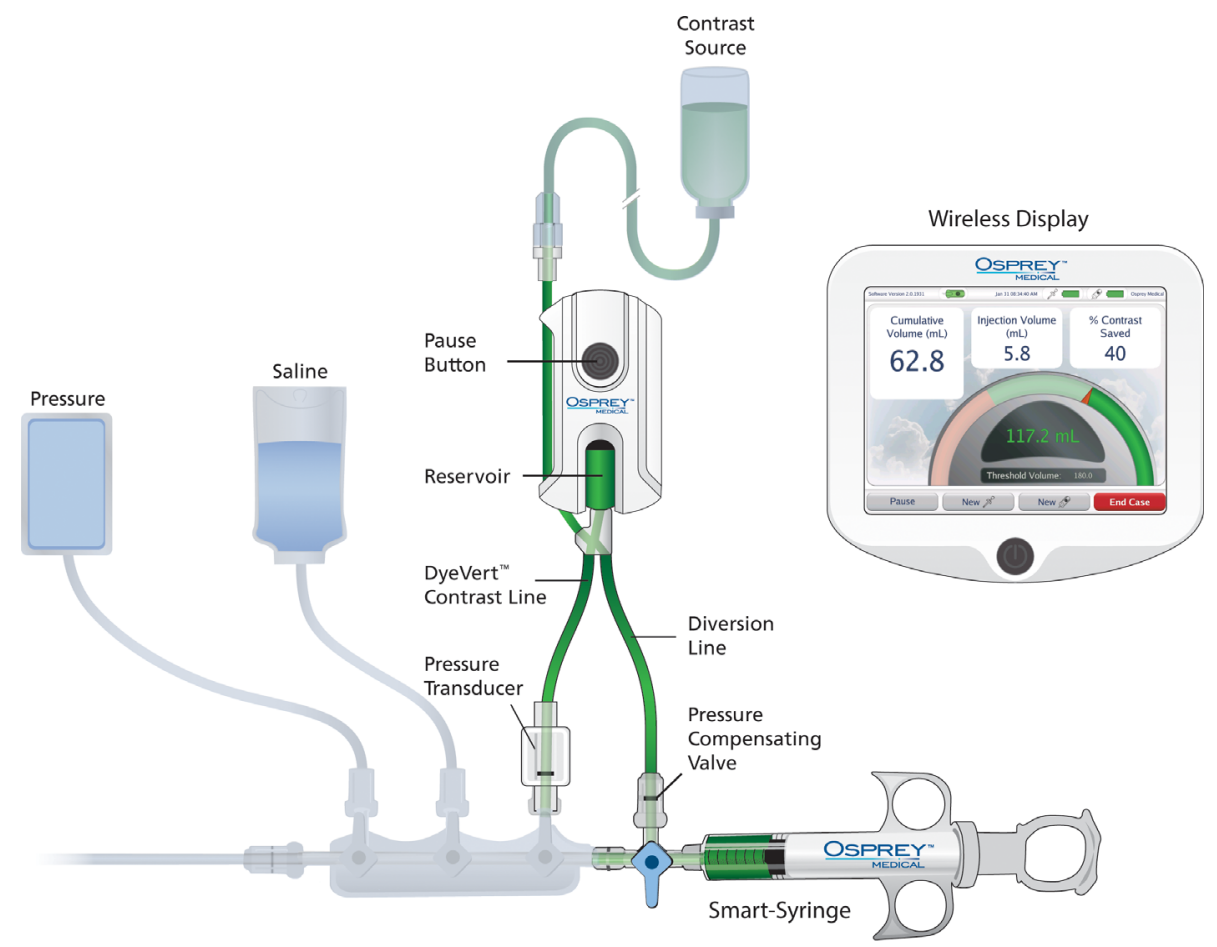

FIGURE 1 The DyeVertTM Plus System [Color figure can be viewed at wileyonlinelibrary.com]

\section{4 | Statistical analysis}

All statistical analyses were performed using SAS software, version 9.4 (SAS Institute Inc., Cary, NC, USA). The primary endpoint analysis was based on the population of evaluable subjects and used a one sample $t$-test against a fixed null hypothesis. Successful rejection of the null hypothesis will demonstrate that the percentage of CMV saved is statistically greater than $30 \%$. The expected percentage of CMV saved was estimated to be at least $35 \% \pm 16 \%$; therefore, a sample size of 100 evaluable subjects was estimated to be required (80\% power, one-sided 0.025 alpha). To account for attrition, 114 subjects were enrolled.

Subjects were deemed unevaluable for primary endpoint analysis if any of the following criteria were met; the DyeVert Reservoir was inadvertently turned off due to user error for $>1$ injection, the DyeVert Reservoir was turned off for $>1$ injection due to an $A E$ not related to the device, the display did not provide contrast accounting details at the end of a case due to device deficiency, a contrast accounting error due to user error occurs for $>1$ injection as defined in the procedure case report form, or the treating physician determines that a Bluetooth disconnection occurred resulting in inaccurate contrast accounting.

All available data were used for the secondary endpoints.

\section{3 | RESULTS}

A total of 114 subjects were enrolled between July through December 2017. Patient and procedure characteristics are listed in Table 1. Baseline eGFR was $43 \pm 11 \mathrm{~mL} / \mathrm{min} / 1.73 \mathrm{~m}^{2}$ with 18 (16\%) subjects having a baseline eGFR of $20-30 \mathrm{~mL} / \mathrm{min} / 1.73 \mathrm{~m}^{2}$. Baseline serum creatinine was $1.6 \pm 0.5 \mathrm{mg} / \mathrm{dL}$. Nearly all subjects had history of hypertension (96\%) and 100 (88\%) subjects had three or more comorbidities in addition to chronic kidney disease (CKD). Twentyfive (22\%) subjects had New York Heart Association functional classification of heart failure Stage III (moderate) or IV (severe). Subjects were, on average, at a moderate risk for AKI according to the Mehran risk score.

Most procedures were CAG only (65\%) and were performed using femoral access (63\%) and $6 \mathrm{Fr}$ (73\%) catheters. Mean pre-defined physician $\mathrm{CM}$ threshold per procedure was $122 \pm 50 \mathrm{~mL}$ and were set using criteria of eGFR $x<2$ not to exceed $3^{14}$ in $66 \%$ of procedures, eGFR $x<3.7^{19}$ in $24 \%$ of procedures, and other methods in $11 \%$ (ePRISM or physician discretion). Cath lab staff reported the DyeVert Plus System setup and priming added $3.3 \pm 2.9$ min to procedure preparation.

Staged $\mathrm{PCl}$ was performed in 11 subjects. Post-procedure serum creatinine measurement was obtained in 54 (47\%) subjects as part of routine clinical care. Same-day discharge occurred in 63 (55\%) subjects, 27 (24\%) subjects were discharged the day after the procedure, five (4\%) subjects were discharged 2 days after the procedure and $19(17 \%)$ subjects were discharged three or more days after the procedure.

\section{1 | Primary endpoint}

Nine subjects were excluded from the primary endpoint analysis per the protocol. In eight cases, the DyeVert Plus System was not used for more than one injection during the case due to user or technical error. In one case, the subject met study exclusion criteria for BMI but was inadvertently enrolled in the study. Therefore, 105 subjects were evaluable for the primary endpoint. 
TABLE 1 Patient and procedural characteristics

\begin{tabular}{|c|c|}
\hline \multicolumn{2}{|l|}{ Patient characteristics } \\
\hline Age (years) & $72 \pm 9$ \\
\hline \multicolumn{2}{|l|}{ Gender } \\
\hline Male & $82(72)$ \\
\hline Female & $32(28)$ \\
\hline \multicolumn{2}{|l|}{ Race } \\
\hline Caucasian & $86(75)$ \\
\hline African American & $25(22)$ \\
\hline Asian & $1(1)$ \\
\hline American Indian & $1(1)$ \\
\hline Other & $3(3)$ \\
\hline BMI & $29 \pm 5$ \\
\hline eGFR $\left(\mathrm{mL} / \mathrm{min} / 1.73 \mathrm{~m}^{2}\right)$ & $43 \pm 11$ \\
\hline Serum creatinine (mg/dL) & $1.6 \pm 0.5$ \\
\hline \multicolumn{2}{|l|}{ Comorbidities } \\
\hline Hypertension & $110(96)$ \\
\hline Coronary artery disease & $86(75)$ \\
\hline Prior $\mathrm{PCl}$ & $60(53)$ \\
\hline Diabetes & $60(53)$ \\
\hline Congestive heart failure & $54(47)$ \\
\hline Prior coronary artery bypass graft & $40(35)$ \\
\hline Prior myocardial infarction & $39(34)$ \\
\hline Anemia & $33(29)$ \\
\hline Angina & $30(26)$ \\
\hline Mehran risk score ${ }^{a}$ (using eGFR) & $9.0 \pm 3.9$ \\
\hline \multicolumn{2}{|l|}{ Procedure characteristics } \\
\hline \multicolumn{2}{|l|}{ Vascular access route } \\
\hline Femoral & $72(63)$ \\
\hline Radial & $42(37)$ \\
\hline \multicolumn{2}{|l|}{ Procedure type } \\
\hline CAG only & $74(65)$ \\
\hline $\mathrm{CAG}+\mathrm{PCl}$ & $30(26)$ \\
\hline $\mathrm{PCl}$ only & $10(9)$ \\
\hline \multicolumn{2}{|l|}{ CM type used } \\
\hline Visipaque 320 & $63(55)$ \\
\hline Omnipaque 350 & $20(18)$ \\
\hline Omnipaque 300 & $16(14)$ \\
\hline Isovue & $8(7)$ \\
\hline Visipaque 270 & $7(6)$ \\
\hline Fluoroscopy time (min) & $12.8 \pm 14.4$ \\
\hline \multicolumn{2}{|l|}{ \# Lesions treated } \\
\hline 0 & $74(65)$ \\
\hline 1 & $29(25)$ \\
\hline 2 & $7(6)$ \\
\hline 3 & $2(2)$ \\
\hline 4 & $1(1)$ \\
\hline 5 & $0(0)$ \\
\hline 6 & $1(1)$ \\
\hline \multicolumn{2}{|l|}{ Maximum catheter size (per subject) } \\
\hline $4 \mathrm{~F}$ & $1(1)$ \\
\hline $5 \mathrm{~F}$ & $20(18)$ \\
\hline $6 \mathrm{~F}$ & $79(73)$ \\
\hline
\end{tabular}

(Continues)
TABLE 1 (Continued)

\begin{tabular}{ll} 
Patient characteristics & \\
\hline $7 F$ & $9(8)$ \\
\hline Catheter size (total reported for all cases) & \\
\hline $4 F$ & $1(1)$ \\
\hline $5 F$ & $42(22)$ \\
\hline $6 F$ & $141(73)$ \\
\hline $7 F$ & $10(5)$
\end{tabular}

Data are $\mathrm{n}(\%)$ or mean \pm SD.

${ }^{a}$ Intra-aortic balloon pump use and hypotension were not collected in this study; risk score integers were assumed 0 for these factors.

The mean predetermined CMV threshold in the primary endpoint cohort was $119 \pm 48 \mathrm{~mL}$ (range $40-236 \mathrm{~mL}$ ). The mean CMV attempted was $112 \pm 85 \mathrm{~mL}$ (range 22-681 mL) and mean CMV delivered was $67 \pm 51 \mathrm{~mL}$ (range $12-403 \mathrm{~mL}$ ) resulting in an overall CMV savings of $40.1 \pm 8.8 \%$ (95\% Cl 38.4, 41.8; $P<0.0001)$ per procedure (Figure 2). In 91 (87\%) cases, the CMV delivered was less than the predefined CM threshold.

When the primary analysis was additionally performed using all cases (inclusive of the excluded cases), the overall CMV savings was $39.4 \pm 9.1 \%$ (95\% Cl 37.7, 41.1; $P<0.0001)$ per procedure.

Image quality was maintained in all but one CAG $+\mathrm{PCl}$ case, in which the physician turned off the DyeVert Plus System for one injection for the purpose of obtaining a better image, and then resumed using the DyeVert Plus System for the remainder of the case.

Prespecified subgroup analyses were performed to assess if contrast savings varied within access approach (radial vs. femoral), BMI $\left(<30 \mathrm{~kg} / \mathrm{m}^{2}\right.$ vs. $\left.\geq 30 \mathrm{~kg} / \mathrm{m}^{2}\right)$, physician user, and procedure type (Table 2). While a large average contrast savings was observed in all subgroups, there were significant differences in contrast savings $(P<0.05)$ for subgroups defined by BMI and procedure type, with more savings observed for those with a lower BMI and for diagnostic procedures. Contrast savings also varied significantly between physician users $(P=0.0029)$.

\section{2 | Secondary endpoints}

All enrolled subjects contributed to the secondary endpoint analysis. No DyeVert Plus System-related AEs or cases of contrast-related anaphylaxis were reported. Acute kidney injury $(>0.3 \mathrm{mg} / \mathrm{dL}$ increase in serum creatinine postprocedure through discharge) was reported in 11 subjects for an observed AKI rate of 9.6\% (11/114). The adjusted $\mathrm{AKI}$ rate, including only those subjects with a post-procedure serum

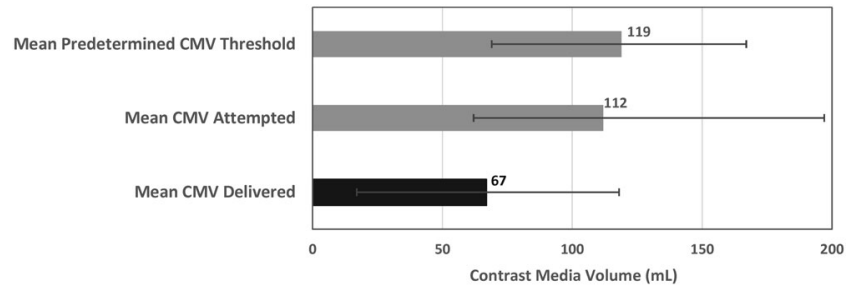

FIGURE 2 Mean contrast media volume attempted (total procedure contrast volume delivered + total procedure contrast volume saved) versus delivered to the patient 
TABLE 2 Contrast volume savings, subgroup analyses

\begin{tabular}{|c|c|c|}
\hline Variable & Percent contrast savings & $P$-value ${ }^{a}$ \\
\hline Access location & & 0.1558 \\
\hline Femoral & $39.2 \pm 8.6(68)$ & \\
\hline Radial & $41.8 \pm 9.0(37)$ & \\
\hline BMI & & 0.0398 \\
\hline$<30$ & $41.6 \pm 8.0(61)$ & \\
\hline$\geq 30$ & $38.0 \pm 9.5(44)$ & \\
\hline Physician & & 0.0029 \\
\hline Physician user 1 & $32.7 \pm 7.5(18)$ & \\
\hline Physician user 2 & $42.3 \pm 5.4(16)$ & \\
\hline Physician user 3 & $41.5 \pm 12.3(15)$ & \\
\hline Physician user 4 & $41.5 \pm 8.2(14)$ & \\
\hline Physician user 5 & $35.9 \pm 5.1(5)$ & \\
\hline Physician user 6 & $44.9 \pm 5.4(5)$ & \\
\hline Physician user 7 & $48.6 \pm 5.0(5)$ & \\
\hline Physician user 8 & $40.7 \pm 7.4(4)$ & \\
\hline Other users ${ }^{b}$ & $40.6 \pm 8.0(23)$ & \\
\hline Procedure type & & 0.0057 \\
\hline Diagnostic only & $41.9 \pm 8.4(70)$ & \\
\hline Diagnostic $+\mathrm{PCl}$ & $37.4 \pm 8.7(25)$ & \\
\hline $\mathrm{PCl}$ only & $34.1 \pm 7.8(10)$ & \\
\hline
\end{tabular}

Data are presented mean \pm std $(n)$.

a $P$-value based on two sample $t$-test or ANOva for subgroup variables with more than two levels.

${ }^{b}$ Physicians with fewer than four cases were combined into Other Users.

creatinine value, was $20.4 \%$ (11/54). Seven AKI events occurred in subjects with baseline eGFR $<30$. Investigators attributed the AKI events to the following causes: five $(4.4 \%)$ were fluid management related (over- or under-diuresis, diuretic use/congestive heart failure), three $(2.6 \%)$ were contrast-related, one was related to a diabetic complication, one was due to a recent prior surgery, and in one case, the cause was unknown.

Using an AKI definition of $\geq 0.5 \mathrm{mg} / \mathrm{dL}$ increase in serum creatinine postprocedure through discharge, six $\mathrm{AKI}$ events were reported for an observed AKI rate of $5.3 \%(6 / 114)$ and an adjusted rate of $11.1 \%(6 / 54)$. Based on an imputed Mehran risk score ${ }^{20}$ (hypotension and use of IABP were assumed to be 0 for all subjects since these variables were not prospectively collected) calculated for this cohort, the predicted risk of AKI (defined as an elevation in serum creatinine of $>0.5 \mathrm{mg} / \mathrm{dL}$ ) for the overall cohort and the patients with follow up serum creatinine data was $14 \%$.

Use of the DyeVert Plus System was associated with a lower observed CMV/eGFR ratios for the study cohort compared to the attempted CMV/eGFR ratios (reflective of the amount of contrast the subject would have been given without the use of DyeVert Plus); therefore, a substantial proportion of subjects moved into lower CMV/eGFR deciles (Figure 3). At lower CMV/eGFR ratios, the use of DyeVert Plus increased the percentage of subjects with ratios $\leq 1$ from $7 \%$ (attempted) to $33 \%$ (actual) and with ratios $\leq 2$ from $42 \%$ (attempted) to $75 \%$ (actual). Conversely, at higher CMV/eGFR ratios, the use of DyeVert Plus reduced the percentage of subjects with ratios $>2$ from $58 \%$ (attempted) to $25 \%$ (actual).

Observed $\mathrm{AKI}$ rates increased with increasing CMV/eGFR ratios (Figure 4), with an observed AKI rate of $0 \%$ for subjects with a CMV/eGFR of $\leq 1$ and $22.2 \%$ for subjects with a CMV/eGFR $>3$ although the number of $\mathrm{AKI}$ events and number of subjects with a high CMV/eGFR was limited.

\section{4 | DISCUSSION}

Our study demonstrates the feasibility of using the DyeVert Plus System to achieve a meaningful reduction in contrast volume delivered to

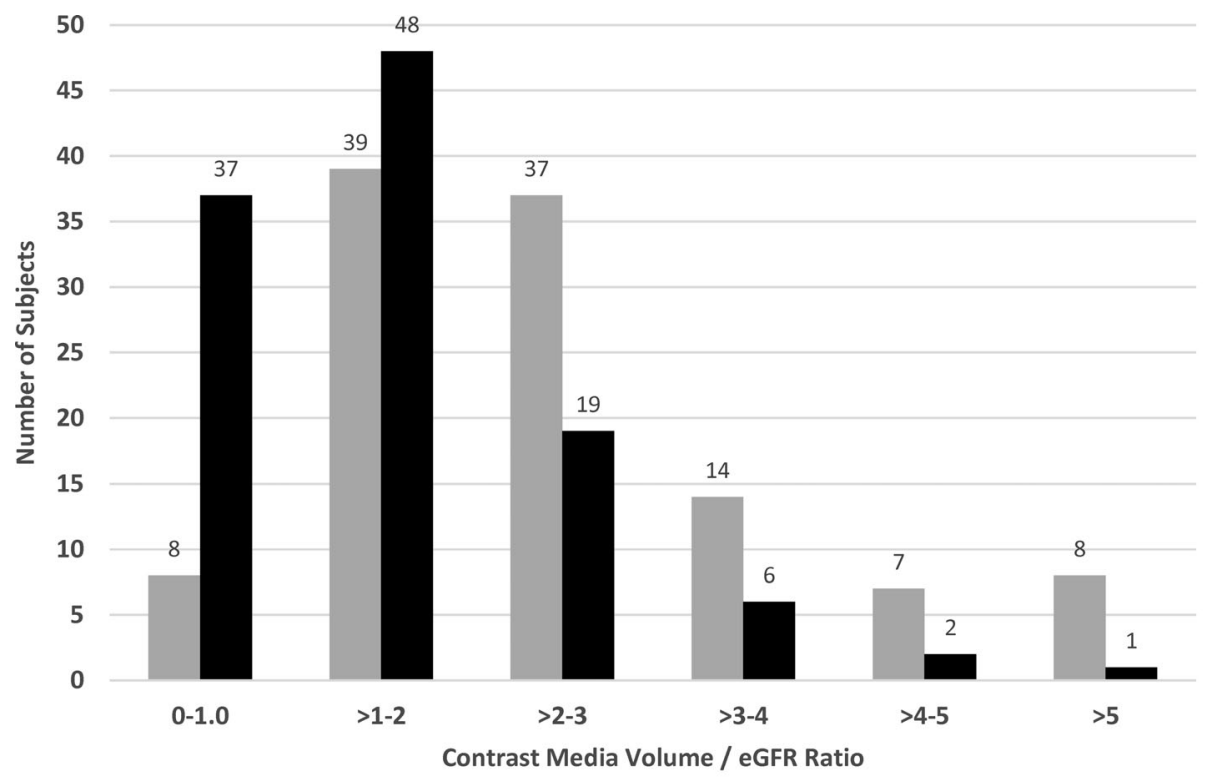

- CMV eGFR Ratio (Attempted)

- CMV eGFR Ratio (Actual)

FIGURE 3 Contrast media volume/baseline estimated glomerular filtration rate ratio attempted (total procedure contrast volume delivered + total procedure contrast volume saved) versus delivered to the patient (actual) using DyeVert Plus 


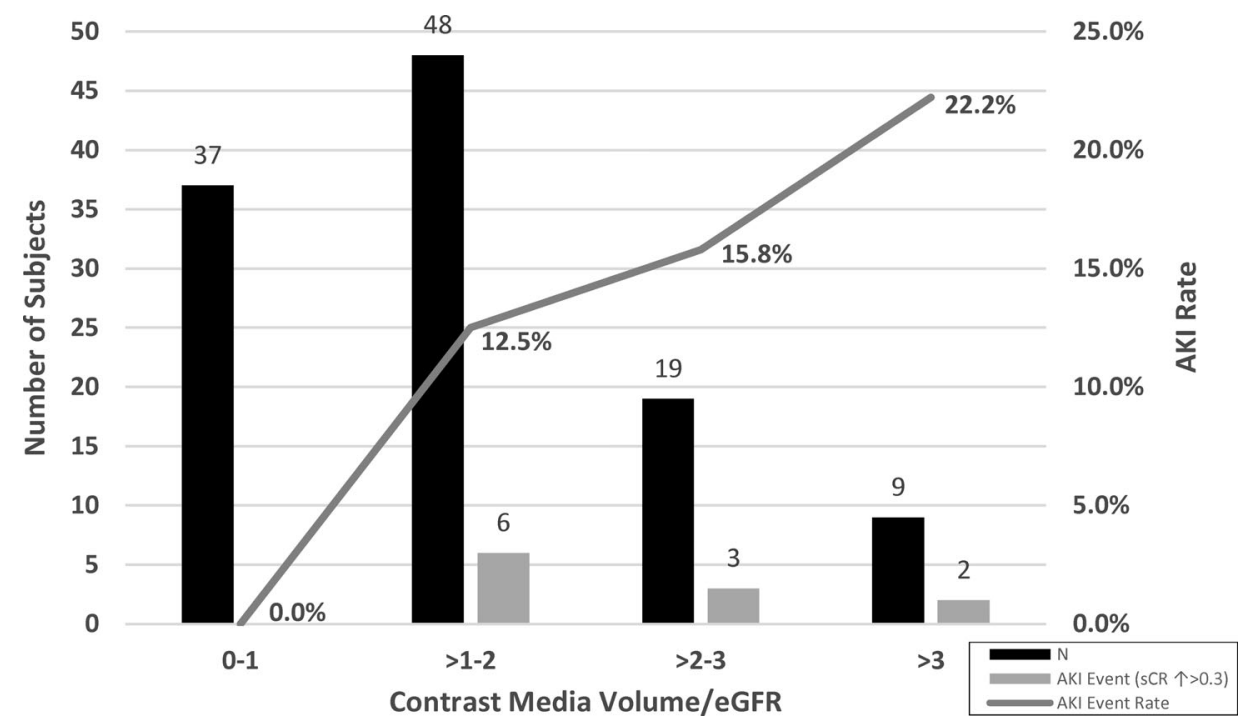

FIGURE 4 Acute kidney injury rates by contrast media volume to baseline estimated glomerular filtration rate ratio

patients undergoing CAG and/or $\mathrm{PCl}$. This was accomplished by a lowering of the CMV-to-eGFR ratio; and thus, a left-ward shift of the renal function-based contrast dose curve. The overall magnitude of the CMV saved was both clinically meaningful and statistically significant. The device was easy to set up and use and no device related complications were observed. In the majority of cases, the CMV delivered was less than the predefined CMV threshold. The observed AKI rate in this study was significantly lower than predicted and adds to the large body of data suggesting that strategies to reduce CMV can result in improved patient outcomes.

Diagnostic CAG and PCls are among the most common procedures using intra-arterial $\mathrm{CM}$ and $\mathrm{AKI}$ remains one of the most common and expensive complications in this population. There is a large body of data suggesting that higher renal function-adjusted CM dose is associated with a higher risk of $\mathrm{AKI}^{4,19-27}$ While the exact threshold for defining renal safety remains debatable, it is clear that patients who receive higher $\mathrm{CM}$ doses are at greater risk of $\mathrm{AKI}$, compared with those who receive lower doses. Furthermore, recent data suggest that collaborative efforts to reduce CMV have been associated with a reduction in the incidence of $A K{ }^{13-15}$

Current $\mathrm{PCl}$ performance standards support careful ascertainment of $\mathrm{CMV}$ in patients undergoing $\mathrm{PCl}$ although this practice remains far from universal. A recent landmark study of over 1.3 million PCls by Amin et al. demonstrated inconsistent and significant variation in contrast use among physicians and minimal reduction in CMV for patients at higher risk for $\mathrm{AKI}^{28}$ In a recent physician survey carried out by SCAl, $40 \%$ of respondents reported estimating CMV without using a measurement technique and $40 \%$ of respondents reported not using $\mathrm{CMV}$ threshold limits for patients at risk of $\mathrm{AKI}{ }^{29}$

The average volume of $\mathrm{CM}$ administered to the patients in this study $(67 \mathrm{~mL}$ ) compares favorably with the volume administered in recent randomized controlled trials (median of $85 \mathrm{~mL}$ reported in the PRESERVE trial $^{30}$ ) or in routine clinical practice (mean of $198 \mathrm{~mL}$ reported by Amin et al. ${ }^{28}$ and $168 \mathrm{~mL}$ reported by Gurm et al. ${ }^{31}$ ). Concurrent with the reduction in CMV, the use of DyeVert Plus resulted in a shift in the actual versus attempted CMV/eGFR ratio. Since the association between $\mathrm{AKI}$ and CMV/eGFR is non-linear, a left-ward shift would be expected to significantly reduce the incidence of AKI. Indeed, none of the patients in whom the CMV/eGFR ratio was less than 1, developed AKI. Further studies to explore the utility of this threshold in high risk patients are warranted.

The DyeVert Plus System probably impacts contrast dose via two related yet equally important mechanisms. First, by minimizing wasted reflux into the aortic root, the system directly reduces the $\mathrm{CMV}$ administered to the patient. Second, by providing direct monitoring of the total CMV delivered to the patient, the system provides direct feedback to the operator and permits modifying the procedure to ensure that the predetermined CM threshold is not exceeded. Finally, the DyeVert Plus System attunes the entire catheterization laboratory to the importance of $\mathrm{CM}$ thresholds and $\mathrm{CMV}$ minimization and potentially helps drive renal safety in the catheterization laboratory.

\section{1 | Study limitations}

We used an objective performance criterion based on published literature instead of a concurrent control group. Additionally, data on $\mathrm{Cl}$ AKI should be construed as hypothesis-generating as postprocedure laboratory data were not available on all patients and $\mathrm{Cl}-\mathrm{AKI}$ was based only on clinically available subject data. AKI events were not available beyond discharge and were not centrally adjudicated. However, the system did significantly reduce administered CM dose, which has been shown to directly correlate with $\mathrm{Cl}-\mathrm{AKI}$ in prior studies.

\section{5 | CONCLUSION}

These data suggest DyeVert Plus System use in CKD subjects undergoing $\mathrm{CAG}$ and/or $\mathrm{PCl}$ procedures results in statistically significant and clinically meaningful CMV savings while maintaining image quality. CMV savings resulted in a meaningful proportion of subjects moving to lower contrast volume-to-eGFR ratios and exploratory analyses 
showed that $\mathrm{Cl}-\mathrm{AKI}$ event rates were significantly lower in subjects with lower contrast volume-to-eGFR ratios.

\section{ACKNOWLEDGMENT}

This study was sponsored and funded by Osprey Medical, Inc.

\section{CONFLICT OF INTEREST}

H Gurm: Consultant-Osprey Medical, Research funding-NIH, Blue Cross Blue Shield of Michigan Foundation.

JL Thomas: Research funding-Osprey Medical.

G Kumar: Consultant-Osprey Medical.

None of the other authors have a direct conflict related to this study.

\section{ORCID}

Hitinder S. Gurm (D) https://orcid.org/0000-0002-1646-0218

Dean J. Kereiakes (1D) https://orcid.org/0000-0003-1086-127X

Gautam Kumar (D) https://orcid.org/0000-0002-4284-846X

\section{REFERENCES}

1. Seeliger E, Sendeski M, Rihal CS, Persson PB. Contrast-induced kidney injury: Mechanisms, risk factors, and prevention. Eur Heart J. 2012; 33(16):2007-2015.

2. Kooiman J, Seth M, Nallamothu BK, Heung M, Humes D, Gurm HS. Association between acute kidney injury and in-hospital mortality in patients undergoing percutaneous coronary interventions. Circ Cardiovasc Interv. 2015;8(6):e002212.

3. Giacoppo D, Madhavan MV, Baber U, et al. Impact of contrast-induced acute kidney injury after percutaneous coronary intervention on short- and long-term outcomes: Pooled analysis from the HORIZONSAMI and ACUITY trials. Circ Cardiovasc Interv. 2015;8(8):e002475.

4. Tsai TT, Patel UD, Chang TI, et al. Contemporary incidence, predictors, and outcomes of acute kidney injury in patients undergoing percutaneous coronary interventions: Insights from the NCDR Cath-PCI registry. JACC Cardiovasc Interv. 2014;7(1):1-9.

5. Neyra JA, Shah S, Mooney R, Jacobsen G, Yee J, Novak JE. Contrast-induced acute kidney injury following coronary angiography: A cohort study of hospitalized patients with or without chronic kidney disease. Nephrol Dial Transplant. 2013;28(6):1463-1471.

6. Naidu SS, Aronow HD, Box LC, et al. SCAI expert consensus statement: 2016 Best practices in the cardiac catheterization laboratory: (Endorsed by the cardiological society of India, and sociedad Latino Americana de Cardiologia intervencionista; affirmation of value by the Canadian Association of interventional cardiology-Association canadienne de cardiologie d'intervention). Catheter Cardiovasc Interv. 2016; 88(3):407-423.

7. Nallamothu BK, Tommaso CL, Anderson HV, et al. ACC/AHA/SCAl/ AMA-Convened PCPI/NCQA 2013 performance measures for adults undergoing percutaneous coronary intervention: $A$ report of the American College of Cardiology/American Heart Association task force on performance measures, the Society for Cardiovascular Angiography and Interventions, the American Medical Associationconvened physician consortium for performance improvement, and the National Committee for quality assurance. Circulation. 2014; 129(8):926-949.

8. Khwaja A. KDIGO clinical practice guidelines for acute kidney injury. Nephron Clin Pract. 2012;120(4):c179-c184.

9. Marenzi G, Assanelli E, Campodonico J, et al. Contrast volume during primary percutaneous coronary intervention and subsequent contrast-induced nephropathy and mortality. Ann Intern Med. 2009; 150(3):170-177.
10. Lauver DA, Carey EG, Bergin IL, Lucchesi BR, Gurm HS. Sildenafil citrate for prophylaxis of nephropathy in an animal model of contrastinduced acute kidney injury. PLoS One. 2014;9(11):e113598.

11. Brown JR, Robb JF, Block CA, et al. Does safe dosing of iodinated contrast prevent contrast-induced acute kidney injury? Circ Cardiovasc Interv. 2010;3(4):346-350.

12. Freeman RV, O'Donnell M, Share D, et al. Nephropathy requiring dialysis after percutaneous coronary intervention and the critical role of an adjusted contrast dose. Am J Cardiol. 2002;90(10): 1068-1073.

13. Brown JR, Solomon RJ, Sarnak MJ, et al. Reducing contrast-induced acute kidney injury using a regional multicenter quality improvement intervention. Circ Cardiovasc Qual Outcomes. 2014;7(5):693-700.

14. Gurm HS, Dixon SR, Smith DE, et al. Renal function-based contrast dosing to define safe limits of radiographic contrast media in patients undergoing percutaneous coronary interventions. J Am Coll Cardiol. 2011;58(9):907-914.

15. Gurm HS, Seth M, Dixon S, Kraft P, Jensen A. Trends in contrast volume use and incidence of acute kidney injury in patients undergoing percutaneous coronary intervention: Insights from blue cross blue shield of Michigan cardiovascular collaborative (BMC2). JACC Cardiovasc Interv. 2018;11(5):509-511.

16. Desch S, Fuernau G, Poss J, et al. Impact of a novel contrast reduction system on contrast savings in coronary angiography-The DyeVert randomised controlled trial. Int J Cardiol. 2018;257:50-53.

17. Sapontis J, Barron G, Seneviratne S, et al. A first in human evaluation of a novel contrast media saving device. Catheter Cardiovasc Interv. 2017;90(6):928-934.

18. Corcione N, Biondi-Zoccai G, Ferraro P, et al. Contrast minimization with the new-generation DyeVert Plus System for contrast reduction and real-time monitoring during coronary and peripheral procedures: First experience. J Invas Cardiol. 2017;29(8):259-262.

19. Laskey WK, Jenkins $C$, Selzer F, et al. Volume-to-creatinine clearance ratio: A pharmacokinetically based risk factor for prediction of early creatinine increase after percutaneous coronary intervention. J Am Coll Cardiol. 2007;50(7):584-590.

20. Mehran R, Aymong ED, Nikolsky E, et al. A simple risk score for prediction of contrast-induced nephropathy after percutaneous coronary intervention: Development and initial validation. J Am Coll Cardiol. 2004;44(7):1393-1399.

21. Raposeiras-Roubin S, Abu-Assi E, Ocaranza-Sanchez R, et al. Dosing of iodinated contrast volume: A new simple algorithm to stratify the risk of contrast-induced nephropathy in patients with acute coronary syndrome. Catheter Cardiovasc Interv. 2013;82(6):888-897.

22. McCullough PA. Contrast-induced acute kidney injury. J Am Coll Cardiol. 2016;68(13):1465-1473.

23. Gurm HS, Seth $M$, Mehran R, et al. Impact of contrast dose reduction on incidence of acute kidney injury (AKI) among patients undergoing PCI: A modeling study. J Invasive Cardiol. 2016;28(4): 142-146.

24. Ogata N, Ikari $Y$, Nanasato $M$, et al. Safety margin of minimized contrast volume during percutaneous coronary intervention in patients with chronic kidney disease. Cardiovasc Interv Ther. 2014;29(3): 209-215.

25. Liu Y, Tan N, Zhou YL, He PC, Luo JF, Chen JY. The contrast medium volume to estimated glomerular filtration rate ratio as a predictor of contrast-induced nephropathy after primary percutaneous coronary intervention. Int Urol Nephrol. 2012;44(1):221-229.

26. Nyman U, Bjork J, Aspelin P, Marenzi G. Contrast medium dose-toGFR ratio: A measure of systemic exposure to predict contrastinduced nephropathy after percutaneous coronary intervention. Acta Radiol. 2008;49(6):658-667.

27. Cigarroa RG, Lange RA, Williams RH, Hillis LD. Dosing of contrast material to prevent contrast nephropathy in patients with renal disease. Am J Med. 1989;86:649-652.

28. Amin AP, Bach RG, Caruso ML, Kennedy KF, Spertus JA. Association of variation in contrast volume with acute kidney injury in patients undergoing percutaneous coronary intervention. JAMA Cardiol. 2017; 2(9):1007-1012.

29. Prasad A, Sohn A, Morales J, et al. Contemporary practice patterns related to the risk of acute kidney injury in the catheterization 
laboratory: Results from a survey of Society of Cardiovascular Angiography and Intervention (SCAl) cardiologists. Catheter Cardiovasc Interv. 2017;89(3):383-392.

30. Weisbord SD, Gallagher M, Jneid H, et al. Outcomes after Angiography with Sodium Bicarbonate and Acetylcysteine. New England Journal of Medicine. 2018;378(7):603-614.

31. Gurm H, Seth M, Dixon S, Kraft P, Jensen A. Trends in contrast volume use and incidence of acute kidney injury in patients undergoing percutaneous coronary intervention. J Am Coll Cardiol. 2018;11(5): 509-511.
How to cite this article: Gurm HS, Mavromatis K, Bertolet B, et al. Minimizing radiographic contrast administration during coronary angiography using a novel contrast reduction system: A multicenter observational study of the DyeVert ${ }^{\mathrm{TM}}$ plus contrast reduction system. Catheter Cardiovasc Interv. 2019;93:

1228-1235. https://doi.org/10.1002/ccd.27935 